\title{
A VISÃO DA EQUIPE DE ENFERMAGEM SOBRE A SISTEMATIZAÇÃO DA ASSISTÊNCIA DE ENFERMAGEM EM UM HOSPITAL DE MÉDIO PORTE
}

\author{
Diego Ferrari ${ }^{1}$; Arlete Eli Kunz da Costa $^{2}$; Luis Felipe Pissaia ${ }^{3}$; Claudete Moreschi ${ }^{4}$
}

\begin{abstract}
Resumo: O objetivo deste estudo é conhecer a visão da equipe de enfermagem de um setor de uma instituição hospitalar sobre a Sistematização da Assistência de Enfermagem. Trata-se de um estudo descritivo de abordagem qualitativa realizado em um hospital de médio porte do Vale do Taquari - RS. Os dados foram coletados entre julho e agosto de 2015 por meio de uma entrevista semiestruturada com oito profissionais da equipe de enfermagem, sendo esses posteriormente analisados em conformidade com a análise de conteúdo. Os dados obtidos apontam que os profissionais têm dificuldades no entendimento e explanação das fases da Sistematização da Assistência de Enfermagem, mas reconhecem a importância delas para a organização, eficiência e eficácia da assistência. O sucesso ou insucesso desse processo depende de fatores essenciais como educação continuada, interesse da equipe, da coordenação e condições mínimas para que a assistência de enfermagem prestada venha a ser sistematizada.
\end{abstract}

Palavras-chave: Processos de Enfermagem. Assistência ao Paciente. Equipe de Enfermagem.

\section{NURSING STAFF'S POINT OF VIEW ABOUT NURSING CARE SYSTEMIZATION IN A MEDIUM-SIZED HOSPITAL}

\begin{abstract}
The objective of this study is recognizing the nursing staff's point of view from a hospital sector about the Nursing Care Systemization. This paper is a descriptive study with a qualitative approach that was held in a medium-sized hospital in Vale do Taquari/RS. The research data was collected between July and August of 2015 by a semi-structured interview with eight professionals from the nursing staff. They were analyzed according to content analysis subsequently. The obtained data show that the professionals have difficulties in understanding and explaining the Nursing Care Systemization but they recognize
\end{abstract}

1 Enfermeiro. Centro Universitário UNIVATES. E- mail: dferrari1@universo.univates.br

2 Enfermeira. Doutora em Ambiente e Desenvolvimento. Docente do Centro de Ciências Biológicas e da Saúde. Centro Universitário UNIVATES. E-mail: arlete.costa@univates.br

3 Enfermeiro. Mestrando em Ensino. Centro Universitário UNIVATES. E-mail: lpissaia@universo.univates.br

4 Enfermeira. Doutora em Ambiente e Desenvolvimento. Centro Universitário UNIVATES. E-mail: clau_moreschi@yahoo.com.br 
its importance to the assistance organization, efficiency and effectiveness. The success or failure of this process depends on essential factors, such as continuing education, team's and coordination interest and minimum conditions in order to systemize the already provided Nursing Care.

Keywords: Nursing Processes. Patient Care. Nursing Staff.

\section{INTRODUÇÃO}

Atualmente na área da saúde, em consonância com demais áreas de prestação de serviços à população, busca-se a excelência pela qualificação e resolutividade das ações compreendidas através dos esforços de equipes de saúde que atuam frente aos processos de saúde e doença de seus clientes (PEREIRA; TOMASI, 2016). Tal excelência, somente ocorre por intermédio da realização da gestão plena do serviço de saúde que engloba medidas administrativas que atuam diretamente à conduta da equipe multiprofissional (QUEIROZ; SILVA, 2016).

$\mathrm{Na}$ área da enfermagem, o planejamento da assistência trata-se de uma exigência legal, que de acordo com a Lei 7498, do Exercício Profissional do Enfermeiro, alínea C, parágrafo I do artigo $8^{\circ}$, é privativo do enfermeiro: "planejamento, organização, coordenação, execução e avaliação dos serviços da assistência de enfermagem" (COFEN, 1986). Reforçando a importância do planejamento da assistência de enfermagem, a Resolução do Conselho Federal de Enfermagem (COFEN) no 358 de 2009, pactua sobre a implementação do Processo de Enfermagem (PE) deve ser realizada de modo deliberado e sistemático em todos os ambientes, públicos ou privados, onde ocorra a atuação de um profissional de Enfermagem (COFEN, 2009).

Neste sentido, a metodologia empregada na área visa à efetividade do cuidado, tratando-se da Sistematização da Assistência de Enfermagem (SAE), considerada como um modelo científico, que cada vez mais vem sendo implantada na prática assistencial, conferindo maior segurança aos pacientes, melhoria da qualidade da assistência e autonomia aos profissionais de enfermagem (TANNURE; PINHEIRO, 2013). A sua implantação nas instituições hospitalares do Brasil baseia-se na necessidade de organização do cuidado a partir de um método sistemático, proporcionando ao enfermeiro a definição do seu espaço de atuação, do seu desempenho no campo de gerência em saúde e da assistência de enfermagem (CASTILHO; RIBEIRO; CHIRELLI, 2009).

A implantação e implementação da SAE em instituições hospitalares segue os moldes preconizados pelo $\mathrm{PE}$, pelo qual a equipe de enfermagem presta o cuidado de forma sistematizada e pautada em princípios científicos comprovados através do registo de sua prática clínica (MANGUEIRA et al., 2012). O PE é constituído por cinco etapas inter-relacionadas, interdependentes e recorrentes, sendo elas, a coleta de dados de enfermagem (ou histórico de enfermagem), diagnóstico de enfermagem, planejamento de enfermagem, implementação e avaliação de enfermagem (COFEN, 2009). 
Diante desta realidade presente nas instituições hospitalares, a SAE compreende a rotina diária das equipes de enfermagem, guiando seus processos de trabalho e conferindo maior confiabilidade na prestação da assistência ao cliente (LEITE et al., 2016). No entanto, a implantação da SAE ainda constitui-se como um desafio dentro das equipes profissionais, pois sua metodologia requer aguçado treinamento nas práticas científicas e mudanças dos modelos tradicionais de atenção à saúde (QUEIROZ; SILVA, 2016).

Para tanto, o presente estudo teve como objetivo conhecer a visão da equipe de enfermagem de um setor de uma instituição hospitalar sobre a Sistematização da Assistência de Enfermagem.

\section{METODOLOGIA}

Trata-se de uma pesquisa de campo, descritiva e exploratória com abordagem qualitativa, realizada com oito profissionais da equipe de enfermagem atuantes em um setor de internação clínica de um hospital geral do Vale do Taquari/RS, Brasil.

A amostra de entrevistados foi estabelecida através de critérios de inclusão de profissionais que atuam há um ano ou mais no referido setor, nos três turnos de trabalho (matutino, vespertino e noturno). Com o auxílio da administração da instituição mapeou-se as pessoas que contemplavam os critérios e dentre os selecionados foram escolhidos aleatoriamente os oito sujeitos, aos quais foi realizado contato telefônico explicando os objetivos da pesquisa e os critérios éticos que a norteiam e convidando-os a participar do estudo.

A coleta de dados ocorreu entre os meses de julho e agosto de 2015, sendo realizada através de horário agendado conforme a disponibilidade dos entrevistados. As entrevistas foram individuais em um espaço na própria instituição, buscando garantir a privacidade e sigilo das informações.

Foram aplicados questionários semiestruturados desenvolvidos pelos próprios pesquisadores, na ocasião os participantes assinaram o Termo de Consentimento Livre e Esclarecido (TCLE) e seus nomes foram preservados por meio do uso de nomes aleatórios de "pássaros". As entrevistas tiveram seus áudios gravados, transcritos e analisados por meio de categorias conforme a Análise de Conteúdo de Bardin (2011).

A pesquisa foi aprovada pela instituição hospitalar alvo de estudo e recebeu parecer de autorização pelo Comitê de Ética em Pesquisa (COEP) do Centro Universitário Univates, através do no 1.154 .581 de 20 de julho de 2015. Foram respeitados os aspectos éticos sobre pesquisas realizadas com seres humanos contemplando a Resolução 466/2012 do Conselho Nacional de Saúde. 


\section{RESULTADOS E DISCUSSÃO}

\section{Caracterizações dos sujeitos da pesquisa}

Quanto à caracterização dos sujeitos da pesquisa, constatou-se que a totalidade dos indivíduos é do sexo feminino. Quanto à sua formação acadêmica seis são Técnicos em Enfermagem e dois graduados em Enfermagem. Quanto à faixa etária, os indivíduos possuíam idade média de 41 anos, ficando entre a faixa dos 31 a 58 anos.

A partir das entrevistas realizadas com os profissionais, as informações foram organizadas e agrupadas em conteúdos compatíveis, resultando em três categorias temáticas: (1) Considerações sobre o conhecimento da equipe de enfermagem sobre a Sistematização da Assistência de Enfermagem; (2) Percepções da equipe de enfermagem quanto a modificações no processo de cuidar com o uso da Sistematização da Assistência de Enfermagem; e (3) As percepções da equipe de enfermagem sobre possíveis dificuldades e limitações na utilização da Sistematização da Assistência de Enfermagem.

\section{Considerações sobre o conhecimento da equipe de enfermagem sobre a Sistematização da Assistência de Enfermagem}

A definição de SAE torna-se importante no contexto da equipe de enfermagem, por estar diretamente ligada aos processos de trabalho desenvolvidos no referido setor, seu domínio facilita a aprendizagem significativa dos envolvidos, capacitando-os mutuamente. Conforme observado a maioria dos profissionais apresentou dificuldades em definir a SAE, bem como os determinantes de sua composição, ao explanar suas ideias demonstravam algum domínio teórico sobre a temática, no entanto nada aprofundado, conforme as falas:

"[...] acho que a SAE é um processo que tem bastante coisas pra fazer, o SAE que temos que fazer a prescrição de enfermagem, mas eu acho que assim ajudou". (Arara)

"A SAE então é a sistematização da assistência de enfermagem, é aonde o enfermeiro faz. uma anamnese do paciente, faz uma prescrição dos cuidados, faz os diagnósticos de enfermagem e prepara isso para que sejam aplicados no paciente". (Sabiá)

Conforme observado em estudos recentes o conhecimento sobre a SAE tornase um determinante relevante em um processo de implantação e implementação da metodologia em um serviço de saúde, pois se relaciona com a prática fragmentada das fases do PE e dificuldades para abdicar dos modelos biomédico e cartesiano (SILVA; MOREIRA, 2011). Verifica-se que atualmente a SAE estrutura-se como uma obrigatoriedade principalmente em ambientes hospitalares, onde ocorre sua implantação sobre bases não estáveis de conhecimento, principalmente pelos profissionais de nível 
médio, ocasionando falhas de conduta e queda na qualidade da assistência oferecida aos clientes (ADAMY; TOSATTI, 2012).

\section{Percepções da equipe de enfermagem quanto a modificações no processo de cuidar com o uso da Sistematização da Assistência de Enfermagem}

Independente dos modelos de trabalho em vigência em instituições hospitalares, no momento em que a SAE é implantada e compactuada com a equipe, as modificações nas rotinas assistenciais são perceptíveis e inerentes a sua necessidade de adaptação. Os entrevistados compreendem as modificações principalmente nos processos de organização do trabalho, considerando a assistência mais efetiva e eficaz perante o cliente, conforme as falas:

"Torna-se fundamental no meu ponto de vista, porque é através dele que a gente pode fazer e prestar um bom trabalho para o paciente". (Aguia)

"[...] vários fatores, mais organizado, organização, tem mais organização nos setores em relação ao $S A E$, por causa do SAE”. (Arara)

Conforme estudos realizados em campo, com participação ativa de pesquisadores em ambientes hospitalares, a utilização da SAE trouxe inúmeros benefícios para a assistência oferecida ao cliente, frisando o aumento significativo na qualidade dos atendimentos, mas também foram observados benefícios voltados à instituição, como a organização do trabalho ocasionando um melhor aproveitamento do tempo pelas equipes (SOUZA; SANTOS; MONTEIRO, 2013). Destacam-se também benefícios direcionados aos profissionais, como a continuidade da assistência à beira do leito, melhoria na qualidade de vida do trabalhador e estimulo em buscar aperfeiçoamento constante na área de atuação (MEDEIROS; SANTOS; CABRAL, 2012).

Ainda em consonância com as informações elencadas, verificou-se o reconhecimento da SAE como um modelo de comunicação interdisciplinar, ou seja, transformando-se como um difusor de conteúdo entre a equipe de enfermagem e a equipe multiprofissional. Conceitua-se como significativo este processo de comunicação interpessoal, por assegurar que as informações dos clientes estejam sob o conhecimento de seus cuidadores e em ambiente seguro, conforme as falas:

"Sim, sempre muda, o paciente em si, ele sempre gosta de receber um pouco mais de atenção e às vezes até através dessa abordagem que a equipe toda se faz com o paciente, a gente tanto visa melhorar o psíquico como mentalmente [...]”. (Beija-Flor)

"[...] porque os profissionais todos passam a conbecer melhor o paciente, ele se obriga, na realidade a fazer uma anamnese completa dele para poder fazer o $S A E$, buscar o bistórico dele, enfim, como é a casa, toda essa parte que ele traz da cultura dele também [...] e todos os profissionais vão saber disso também". (Sabiá) 
A equipe multiprofissional beneficia-se pela $\mathrm{SAE}$, devido à aplicação do $\mathrm{PE}$ que possibilita uma assistência de forma sistematizada, pautada em princípios científicos e passiveis de comunicação interpessoal ocasionada pelo diálogo multidisciplinar criado por meio do modelo (MANGUEIRA et al., 2012). Modelos científicos de implantação da SAE realizam uma reflexão sobre o reconhecimento da modalidade não apenas na prática clínica, mas também como meio teórico de aprendizagem assistencial, colaborando com modelos holísticos e de avaliação integral do ser humano (SILVA et al., 2015).

\section{As percepções da equipe de enfermagem sobre possíveis dificuldades e limitações na utilização da Sistematização da Assistência de Enfermagem}

A reflexão sobre a metodologia utilizada pela SAE torna-se importante para o contexto da equipe de enfermagem, pois seu fluxo pode ser flexível, e os problemas devem ser identificados e superados em sua plenitude. Conforme as informações coletadas a metodologia da SAE não apresenta dificuldades, no entanto sua adesão e eficiência são prejudicadas devido a déficit de profissionais nas equipes e sua desmotivação em procurar entender mais sobre o modelo, conforme os relatos:

"[... falta de pessoal, falta de tempo mesmo e a implementação, quando se tem limitado pela falta de, de recurso humano, mão de obra que eles não acabam tendo esse tempo e eu acho também que eles não têm muito conhecimento do que é o $S A E$, eles não vão no prontuário, ler a prescrição do enfermeiro para colocar ela em prática". (Sabiá)

"[...] a mão de obra pra trabalhar a gente falta muito, falta estetoscópio, falta, falta termômetro, às vezes falta material pra trabalhar, falta bastante assim, no caso de primeiros socorros, sinais vitais, isso tá deixando muito a desejar, que o pessoal se atrasa, muito de verificar sinais vitais porque não tem material [...]". (Bem-Te-Vi)

Em um estudo semelhante de caráter descritivo e exploratório realizado no setor de Pediatria do Hospital Universitário Clemente Faria - HUCF, da cidade de Montes Claros, Minas Gerais, em 2013, as principais dificuldades encontradas pela equipe de enfermagem na implementação da SAE, recebendo destaque a sobrecarga de trabalho, o número de funcionários inadequado para a demanda da assistência, bem como a carência na estrutura física e a ausência de materiais (SILVA et al., 2014). Considerando que cada estabelecimento de saúde possui suas particularidades, para que o método seja utilizado em sua plenitude com metas possíveis de ser alcançadas, a equipe de enfermagem deve analisar as facilidades e dificuldades que encontrarem antes e após a sua adesão e refletir quanto a sua própria resolutividade (SILVA et al., 2015). 


\section{CONSIDERAÇÕES FINAIS}

Constatou-se que no setor estudado não são feitos todos os passos da SAE devido às dificuldades elencadas, verificou-se também por parte da equipe um conhecimento escasso sobre a metodologia, o que representa a necessidade de um maior aprofundamento no tema. Compreendeu-se que a SAE traz benefícios ao cliente e também à equipe, pois organiza os processos de trabalho e facilita a prestação de assistência, havendo consequentemente um maior reconhecimento dos profissionais de enfermagem.

Apresentaram-se várias barreiras para a implementação efetiva da SAE, podendo torná-la um processo desestimulador, apenas persistindo como uma atividade meramente burocrática e de responsabilidade de alguns profissionais. Os profissionais compreendem que para o sucesso desse método é necessário uma estrutura mínima em termos de recursos humanos, físicos, de tempo e interesse da equipe de enfermagem.

Para tanto se considera que o sucesso na utilização da SAE não depende somente de estímulos externos à instituição ou a equipe, e sim de fatores intrínsecos aos próprios sujeitos assistenciais. Nestes processos classificam-se como essenciais a realização de educação continuada, estímulo das equipes em buscar seu próprio conhecimento e reorganizar os fluxos de trabalho para assim iniciar o trabalho através da metodologia da SAE.

\section{REFERÊNCIAS}

ADAMY, E. K.; TOSATTI, M. Sistematização da Assistencia de Enfermagem no período Peri operatório: Visão da Equipe de Enfermagem. Rev Enferm UFSM. v. 2, n. 2, n. 300-310, Mai/Ago, 2012.

BARDIN, L. Análise de conteúdo. 70. ed. Lisboa/Portugal, LDA, 2011.

CASTILHO, N. C.; RIBEIRO, P. C.; CHIRELLI, M. Q. A implementação da sistematização da assistência de enfermagem no serviço de saúde hospitalar do Brasil. Texto contexto - enferm. v. 18, n. 2, p. 280-9, Abr/Jun, 2009.

CONSELHO FEDERAL DE ENFERMAGEM. Resolução no 358, de 15 de outubro de 2009. Dispõe sobre a Sistematização da Assistência de Enfermagem e a implementação do Processo de Enfermagem em ambientes, públicos ou privados, em que ocorre o cuidado profissional de enfermagem, e dá outras providências. Disponível em: <http://www.cofen.gov. br/resoluocofen-3582009_4384.html>. [Acesso em: 05 de set. 2016].

CONSELHO FEDERAL DE ENFERMAGEM. Lei n 7.498, de 25 de junho de 1986.

Dispõe sobre a regulamentação do exercício da Enfermagem e dá outras providências.

Disponível em: <http://www.cofen.gov.br/lei-n-749886-de-25-de-junho-de-1986_4161.html〉. [Acesso em: 05 set. 2016]. 
LEITE, M. T. et al. Gestão do cuidado na estratégia saúde da família: revisão narrativa.

Revista de Atenção à Saúde (antiga Rev. Bras. Ciên. Saúde), v. 14, n. 48, p. 106-115, 2016.

MANGUEIRA, S. O. et al. Implantação da sistematização da assistência de enfermagem: opinião de uma equipe de enfermagem hospitalar. Enfermagem em Foco. v. 3, n. 3, p. 135138, 2012.

MEDEIROS, A. L.; SANTOS, S. R.; CABRAL, R. W. L. Sistematização da assistência de enfermagem na perspectiva dos enfermeiros: uma abordagem metodológica na teoria fundamentada. Rev Gaúcha Enferm. v. 33, n. 3, p. 174-181, 2012.

PEREIRA, B. S.; TOMASI, E. Instrumento de apoio à gestão regional de saúde para monitoramento de indicadores de saúde. Epidemiologia e Serviços de Saúde, v. 25, n. 2, p. 411-418, 2016.

QUEIROZ, N. T. B.; SILVA, V. C. F. A educação permanente no cotidiano das equipes da Estratégia Saúde da Família. IV Jornada de Pesquisa e Iniciação Científica do UNIFESO (JOPIC-2014), v. 1, n. 1, 2016.

SILVA, C. F. M. et al. Sistematização da assistência de enfermagem: percepção dos enfermeiros. Rev Enferm UFPI. v. 4, n. 1, p. 47-53, Jan/Mar, 2015.

SILVA, F. R. et al. Implementação da Sistematização da Assistência de Enfermagem: Dificuldades e Potencialidades. Revista da Universidade Vale do Rio Verde. Três Corações. v. 12, n. 2, p. 580-590, Ago/Dez, 2014.

SILVA, M. M.; MOREIRA, M. C. Sistematização da assistência de enfermagem em cuidados paliativos na oncologia: visão dos enfermeiros. Acta Paul Enferm. v. 24, n. 2, p. 172-8, 2011.

SOUZA, M. F. G.; SANTOS, A. D. B.; MONTEIRO, A. I. O processo de enfermagem na concepção de profissionais de Enfermagem de um hospital de ensino. Rev Bras Enferm. Brasília, v. 66, n. 2, p. 167-73, 2013.

TANNURE, M. C.; PINHEIRO, A. M. SAE - Sistematização da assistência de enfermagem: guia prático. 2. ed. Rio de Janeiro: Guanabara Koogan; 2013. 